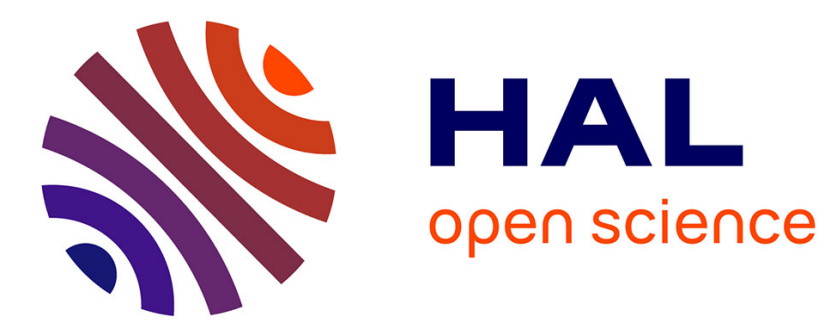

\title{
Input referred noise of magneto-resistor based current conditioners
}

Gilles Allègre, Maria Timofeeva, J. Thomas, Stéphane Flament, Didier Robbes

\section{To cite this version:}

Gilles Allègre, Maria Timofeeva, J. Thomas, Stéphane Flament, Didier Robbes. Input referred noise of magneto-resistor based current conditioners. Noise and Fluctuations (ICNF), 2013 22nd International Conference on, Jun 2013, Montpellier, France. 10.1109/ICNF.2013.6578902 . hal-01110622

\section{HAL Id: hal-01110622 \\ https://hal.science/hal-01110622}

Submitted on 28 Jan 2015

HAL is a multi-disciplinary open access archive for the deposit and dissemination of scientific research documents, whether they are published or not. The documents may come from teaching and research institutions in France or abroad, or from public or private research centers.
L'archive ouverte pluridisciplinaire HAL, est destinée au dépôt et à la diffusion de documents scientifiques de niveau recherche, publiés ou non, émanant des établissements d'enseignement et de recherche français ou étrangers, des laboratoires publics ou privés. 


\title{
Input referred noise of magneto-resistor based current conditioners
}

\author{
G. Allegre ${ }^{1,2}$, M. Timofeeva ${ }^{1}$, J. Thomas ${ }^{3}$, S.Flament ${ }^{1,3}$, D. Robbes ${ }^{1,4}$ \\ ${ }^{1}$ GREYC UMR 6072, ${ }^{2}$ IUT de Caen, ${ }^{3}$ ENSICAEN, ${ }^{4}$ Université de Caen Basse Normandie \\ 6 Bd Marechal Juin Caen, France \\ gilles.allegre@unicaen.fr, stephane.flament@ensicaen.fr
}

\begin{abstract}
This contribution presents, in a reverse engineering like approach, the noise measurements and analysis of two commercially available magneto-resistive currents sensors respectively based on GMR (NVE Inc.) and AMR (SENSITEC GmbH) Wheatstone bridges. Comparison is done with the ultimate aim of designing low noise current conditioners for mini search coils based, magnetometers with galvanic insulation useful for further developments.
\end{abstract}

Keywords-Transconductance amplifier; current detector; current noise; AMR and GMR bridges; hight conductance current conditioning;

\section{INTRODUCTION}

In the frame of an industrial research on miniature search coil magnetometers and conditioning units we pointed that the usual, high input impedance, differential voltage amplifier, has no true equivalent current amplifier, having both high admittance and differential inputs. As a matter of fact, one usually designs such current or transimpedance amplifiers [1,2] using op-amps, feedback loops and "virtual ground" input ports, which cannot be qualified as true current conditioner having actual high conductance inputs, whose optimal configuration is a short circuit. Commercially available current sensing devices (CSD) exhibiting these features, but in nondifferential arrangement, were evaluated especially to better know their noise properties when used as transimpedance amplifiers. Obtained data, that lack in literature though of primary interest, could be added to specifications sheets of such devices. This would help both electronic designers of such circuits to improve their performances, and general design of low noise applications that need such differential transimpedance (or current) amplifiers to perform low noise, high linearity and dynamic range current read out conditioning units. Current sensors based on Giant Magneto Resistors (GMR) such as those provided by NVE Inc. are designed for low current applications. Those from SENSITEC GmbH are using Anisotropic Magneto Resistors (AMR) and designed for rather high current applications, (tens of $A$ ).

For both devices we were able to measure and explain the input referred noise model of both devices which is not provided by the companies. Comparison between SENSITEC and NVE current sensors shows that the input noise level can be strongly reduced by increasing the magnetic transfer between the input current and the GMR or AMR bridge. Optimized coupling between input strip and AMR or GMR bridge is then discussed to achieve magnetometers performance obtained with OpAmps based conditioners.

\section{TRANSFER FUNCTION AND INPUT REFERRED NOISE CURRENT}

\section{A. GMR Bridge as an open loop current sensor}

NVE Inc. sells a GMR Wheastone bridge including an on chip current strip efficiently coupled to the bridge while the latter is shielded from other external magnetic fields sources (Fig. 1) [3]. The transimpedance of this current sensing device (CSD) is equal to $0.08 \mathrm{mV} / \mathrm{mA}$ per volt of bridge $\mathrm{DC}$ bias and its bandwidth is given equal to $100 \mathrm{kHz}$. The chip does not include any voltage differential instrumentation amplifier connected to the bridge and the input admittance is rather large (typical value equal to $4 \mathrm{~S}$ ) so that the noise is dominated by the Johnson and low frequency excess noise of the GMR resistors.

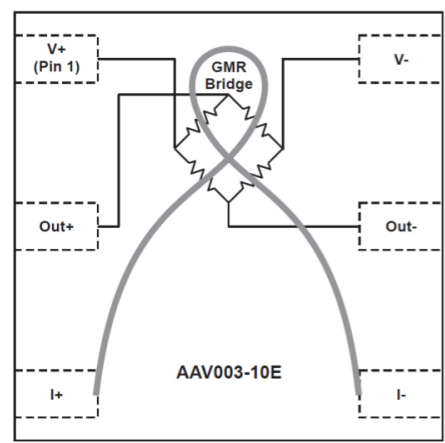

Fig. 1. Functional diagram of the GMR current sensor from NVE Inc.

By connecting a low noise instrumentation amplifier (INA 163 from Texas Instruments [4]) to the GMR bridge and then measuring both the transfer function of the device and the output noise, we obtained an input referred noise current floor $i_{n \text { eq }}$ equal to $9.5 \mathrm{nA} / \sqrt{\mathrm{Hz}}$. This value lies in the upper white noise range $[7 \mathrm{nA} / \sqrt{\mathrm{Hz}} ; 9.5 \mathrm{nA} / \sqrt{\mathrm{Hz}}]$ that can be calculated taking into account the Johnson noise of the bridge resistance (whose value is given in the datasheet to range from $5.5 \mathrm{k} \Omega$ to $8.5 \mathrm{k} \Omega$ ), the noise contribution of the INA 163 (typical input voltage and current noises of $1 \mathrm{nV} / \sqrt{\mathrm{Hz}}$ and 
$0.8 \mathrm{pA} / \sqrt{\mathrm{Hz}}$ respectively, output noise of $60 \mathrm{nV} / \sqrt{\mathrm{Hz}}$, gain equal to 11 providing a bandwidth larger than $1 \mathrm{MHz}$ ) and the transimpedance of $1.6 \mathrm{~V} / \mathrm{A}$ (for a $20 \mathrm{~V} \mathrm{DC}$ Bias). It is to be noticed that up to $100 \mathrm{kHz}$, the noise is dominated by the excess low frequency noise of the GMR. (Fig. 2).

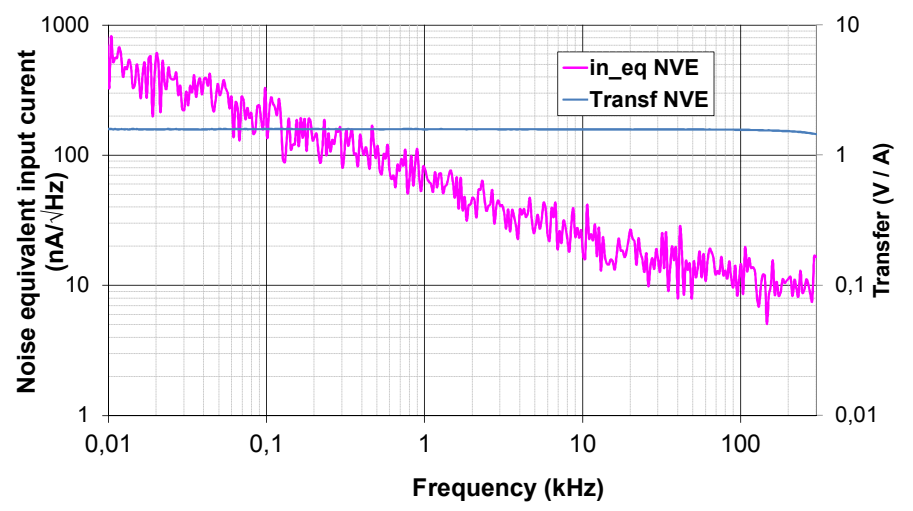

Fig. 2. Tranfer function and input referred noise current of the GMR current sensor from NVE Inc.

\section{B. Closed loop current sensor based on an AMR Bridge}

The AMR based CSD provided by SENSITEC GmbH, are designed for high current applications, (tens of A) [5]. In this case an AMR Wheatstone bridge is operating in a closed loop mode including a high gain voltage amplifier connected to the AMR bridge output (see Figs. 3\&4): the input path (primary conductor) is a few turns coil and the return path (compensation conductor) is, here too, an on chip current strip. By measuring both the transfer function of the device and the output noise, we deduce an input referred noise current floor $\mathrm{i}_{\mathrm{n} \text { eq }}$ equal to $3.6 \mu \mathrm{A} / \sqrt{\mathrm{Hz}}$ (Fig. 5) which is much higher than the NVE CDS one. This value can be explained by the small value of the intrinsic transimpedance between the input path and the AMR bridge, which is not provided by SENSITEC but which was investigated as detailed in section III. The transimpedance $\mathrm{T}_{\mathrm{AMR}}$ of the SENSITEC CSD is around $0.5 \mathrm{~V} / \mathrm{A}$ as given in the datasheet. It is to be noticed that low frequency noise of AMR is in excess only up to $130 \mathrm{~Hz}$.

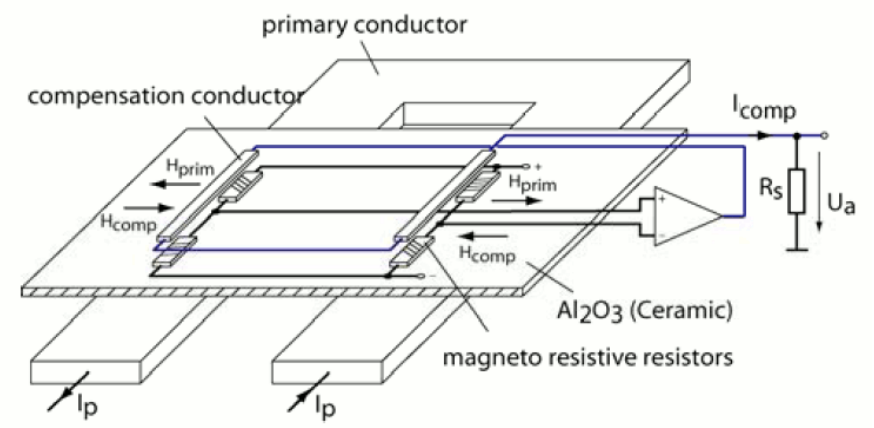

Fig. 3. Design and functional diagram of the AMR based current sensor from SENSITEC GmbH [6].

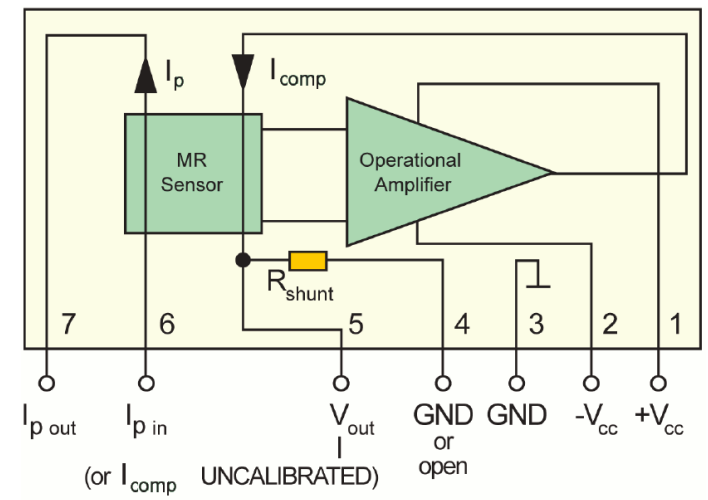

Fig. 4. Available pins for the CMS5005 SENSITEC current sensor. Bridge output is available only by connecting additionnal probe contacts to the sensor's board.

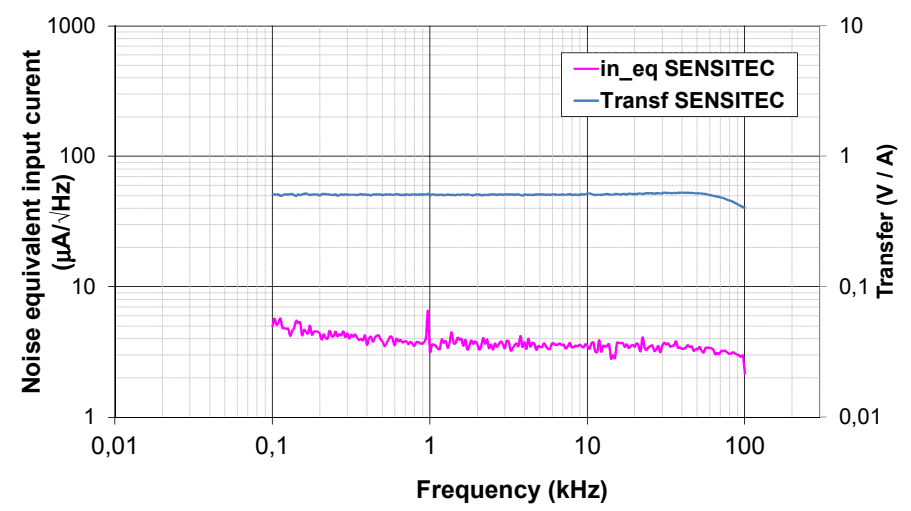

Fig. 5. Tranfer function and input referred noise current of the AMR current sensor from SENSITEC GmbH.

\section{COMPARISON OF NOISE PERFORMANCE OF GMR AND AMR BRIDGES}

For the NVE CSD, we measured an input referred noise that is dominated by the excess low frequency noise of the GMR (Fig. 2) up to $100 \mathrm{kHz}$. This is in accordance with Stutzke et al [7] characterization of such GMR based sensors. In the case of the SENSITEC CSD, one needs to take into account that the bridge is not used the same way (closed loop against open loop) and one needs to investigate whether the noise is mainly due to the AMR bridge noise or if other noise contributions, like the amplifier ones, have to be taken into account. This requires a better knowledge of the device specifications and namely the evaluation of the transimpedance $\mathrm{T}_{\text {In }}$ between the input path and the AMR bridge as well as the transimpedance $\mathrm{T}_{\text {Comp }}$ between the return path and the AMR bridge. From Fig. 4 (with $\mathrm{R}_{\text {shunt }}$ connected to ground), one obtains:

$$
\mathrm{V}_{\text {out }}=A_{\mathrm{v}} \cdot\left(\mathrm{T}_{\mathrm{In}} \cdot \mathrm{I}_{\mathrm{p}}-\mathrm{T}_{\mathrm{Comp}} \cdot \mathrm{I}_{\mathrm{comp}}\right)
$$$$
\text { where } \quad I_{\text {comp }}=\frac{V_{\text {out }}}{R_{\text {shunt }}} \text {. }
$$

Thus for sufficiently large values of the gain (i.e. below the closed loop cut off frequency), we have: 


$$
\mathrm{V}_{\text {out }}=\mathrm{R}_{\text {shunt }} \cdot \frac{\mathrm{T}_{\text {In }}}{\mathrm{T}_{\text {Comp }}} \cdot \mathrm{I}_{\mathrm{p}}=\mathrm{T}_{\text {AMR }} \cdot \mathrm{I}_{\mathrm{p}}
$$

For $\mathrm{R}_{\text {shunt }}=100 \Omega, \mathrm{T}_{\mathrm{AMR}}$ is given equal $0.5 \mathrm{~V} / \mathrm{A}$ in the datasheet, which value was experimentally verified to be valid up to $100 \mathrm{kHz}$. This also means that the ratio $\frac{\mathrm{T}_{\text {Comp }}}{\mathrm{T}_{\mathrm{In}}}$ is equal to 200 .

Assuming that the white noise at the amplifier stage input is dominated by the voltage noise $e_{n}$ of the AMR Bridge resistance, which is $4.1 \cdot \sqrt{\mathrm{R}_{\mathrm{AMR}}(\mathrm{k} \Omega)} \mathrm{nV} / \sqrt{\mathrm{Hz}}$ at $300 \mathrm{~K}$, the transimpedance can been obtained by the measurement of the output voltage noise $\mathrm{V}_{\text {out, }}$ through the relation:

$$
\mathrm{T}_{\mathrm{In}}=\mathrm{T}_{\mathrm{AMR}} \cdot \frac{\mathrm{e}_{\mathrm{n}}}{\mathrm{V}_{\text {Out,n }}}=\frac{\mathrm{e}_{\mathrm{n}}}{\mathrm{i}_{\mathrm{n}_{\mathrm{n}} \text { eq }}}
$$

From the output noise measurement and a measured value of $2.2 \mathrm{k} \Omega$ for $\mathrm{R}_{\mathrm{AMR}}$, we obtained $\mathrm{T}_{\mathrm{In}}=1.7 \mathrm{mV} / \mathrm{A}$. Besides, the noise level at the amplifier stage input was measured separately using a low noise instrumentation amplifier connected in parallel to this amplifier stage. The measured value was consistent with the noise level of an AMR bridge resistance of $2.2 \mathrm{k} \Omega$ and thus with the assumption that the white noise was dominated by the Johnson noise of this AMR bridge.

The values of $T_{\text {In }}$ and $T_{\text {Comp }}$ were checked using the following method. Considering the design of both input and return paths, we assumed that the capacity of each path was of negligible effect so that the output expression (1), relating the output voltage to the input and return currents, was taken as valid over the whole bandwidth of this study. Over the SENSITEC CSD bandwidth, $I_{p}$ and $I_{\text {comp }}$ currents and the bridge voltage $V_{B}$ are all in phase. Above the cut-off frequency, there is a frequency dependent phase difference between these three terms. Fig. 6 shows typical waveforms obtained at $150 \mathrm{kHz}$ using probes and a differential amplifier (NF ELECTRONIC INSTRUMENTS 5305) to obtain bridge voltage $\mathrm{V}_{\mathrm{B}}$.

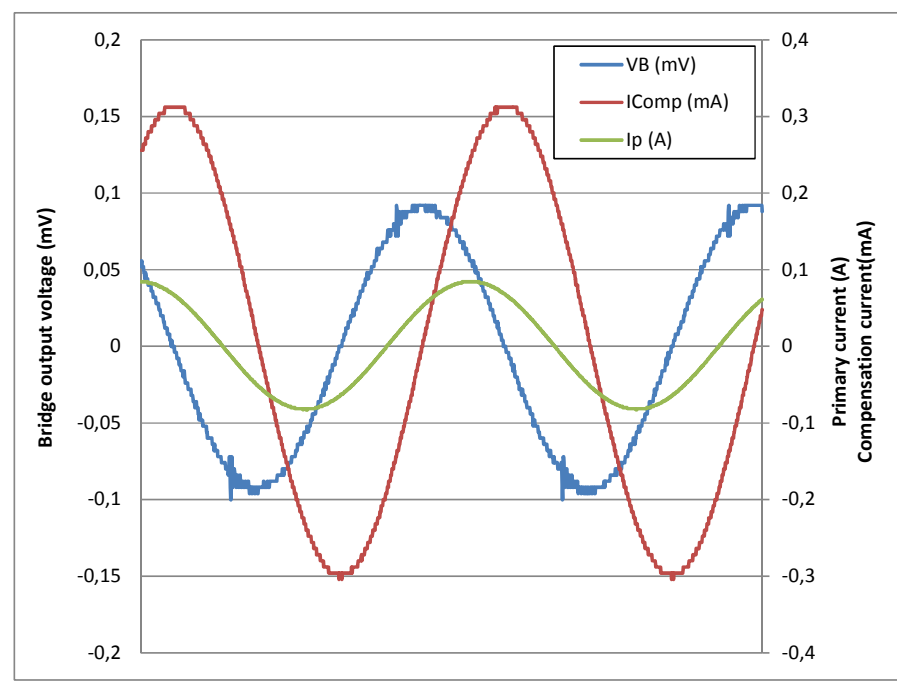

Fig. 6. Waveforms of bridge voltage and input and return current at $150 \mathrm{kHz}$
From these waveforms, the two values $T_{\text {In }}$ and $T_{\text {Comp }}$ were accurately calculated using a two variable linear regression. We obtained very precisely (relative deviation of $1 \%$ ) $\mathrm{T}_{\mathrm{In}}=1.778 \mathrm{mV} / \mathrm{A}$ and $\mathrm{T}_{\text {Comp }}=0.3832 \mathrm{~V} / \mathrm{A}$. These values lead to a ratio $\frac{T_{\text {Comp }}}{T_{\text {In }}}$ equal to 213 which is consistent with the preceding value 200 deduced from datasheet analysis. The value for $T_{\text {In }}$ is very close to the value $T_{\text {In }}=1.7 \mathrm{mV} / \mathrm{A}$ deduced from noise measurements.

If the SENSITEC CSD was operated in a simpler way i.e. in an open loop mode and using the return path as the input path and the bridge output as the NVE device does, the transimpedance could be largely increased and thus the input referred noise current largely improved. More precisely, the transimpedance of the SENSITEC CSD could be increased by a factor of 200 just by using the return path as input path, since the transimpedance of the return path was evaluated 200 larger that the input path one. One can thus expect an input referred white current noise reduced down to $\mathrm{I}_{\mathrm{p}, \mathrm{n}}=16 n \mathrm{n} / \sqrt{\mathrm{Hz}}$ i.e. a value similar to the NVE CSD. The same level would be obtained in the closed loop mode if both input and return paths had the same geometry and the same value of transimpedance equal to $\mathrm{T}_{\text {Comp. }}$. Furthermore the noise level over the full bandwidth would be less than the NVE CSD ones since the AMR excess noise is usually higher that the GMR one [7]. We verified this analysis by measuring the noise at the bridge output with the low noise 5305 amplifier. It was then possible to calculate the equivalent current noise level at the return path level by dividing this voltage noise by the transimpedance $\mathrm{T}_{\text {Comp }}$ of the return path. The obtained level is slightly above the preceding white noise level due to the noise of the amplifier

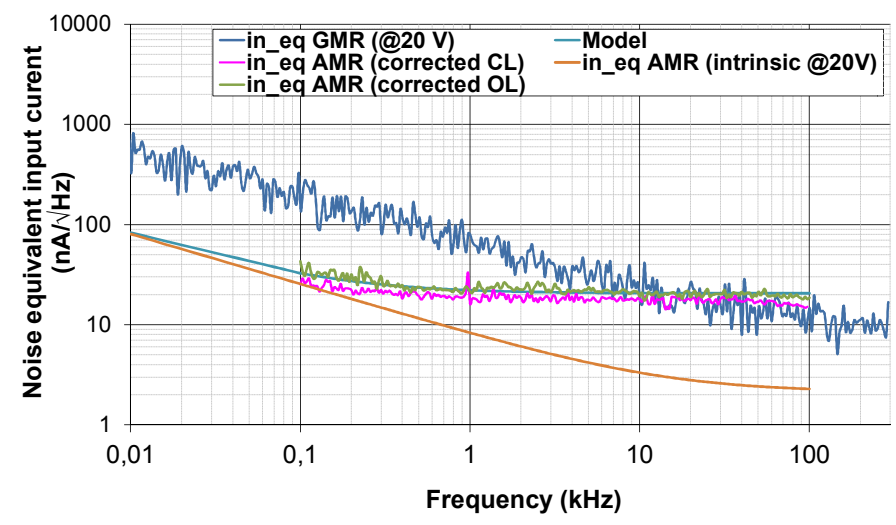

Fig. 7. Actual input referred current noise of NVE Inc GMR sensor (with a bridge DC biasing of $20 \mathrm{~V}$, Blue curve 'in-eq GMR @20 V'); noise of a SENSITEC AMR sensor operating in closed loop and including an improved input path having a magnetic coupling as efficient as the return path thus leading to the same value of $\mathrm{T}_{\text {In }}$ and $\mathrm{T}_{\text {Comp }}$ (pink curve 'in eq AMR (corrected CL)'); noise of a SENSITEC AMR sensor operating in an open loop mode using the return path as input path connected to instrumentation amplifier (green curve line 'in eq AMR (corrected OL)'); noise model deduced of a SENSITEC AMR sensor operating in an open loop (white blue curve 'Model') from which the low frequency AMR excess noise is modeled; intrinsic noise of a SENSITEC AMR sensor in closed loop with a $20 \mathrm{~V}$ DC biasing instead of the current 2.7 V DC Biasing and including an improved input path (orange curve 'in_eq AMR (intrinsic @ 20 V)'). 
which provides an equivalent current white noise of $I_{p, n}=720 \mathrm{fA} / \sqrt{\mathrm{Hz}}$. The noise curve was modeled by the sum of a white noise contribution and a $1 / \mathrm{f}$ excess noise contribution. This excess noise was taken into account for evaluating the achievable input referred current noise when the DC biasing of the AMR bridge is increased. The transimpedance can effectively be further improved by increasing the DC bias of the AMR bridge from $2.7 \mathrm{~V}$ (which is the present value of DC bias mentioned in the datasheet) to $20 \mathrm{~V}$ (which is the upper value used for the NVE Inc. GMR bridge). This would result in a further reduction of the input referred white current noise contribution but would have no effect on the $1 / \mathrm{f}$ noise contribution. Fig. 7 provides on the same caption the available noise levels of GMR and AMR sensors for different operating conditions.

\section{IMPROVEMENT OF MR SENSORS FOR APPLICATION TO MAGNETOMETRY}

AMR and GMR CSD can also be used as transimpedance amplifiers. They were evaluated in the frame of an industrial research on miniature search coil magnetometers and conditioning units. The search coils operating in flux mode and having a current read out can be connected either to AMR or GMR transimpedance amplifiers or to OpAmps based transimpedance amplifiers. An example of such transimpedance amplifier is an instrumentation amplifier structure where the variable gain ports are used for connecting the search coils instead of a resistor fixing the gain [8].

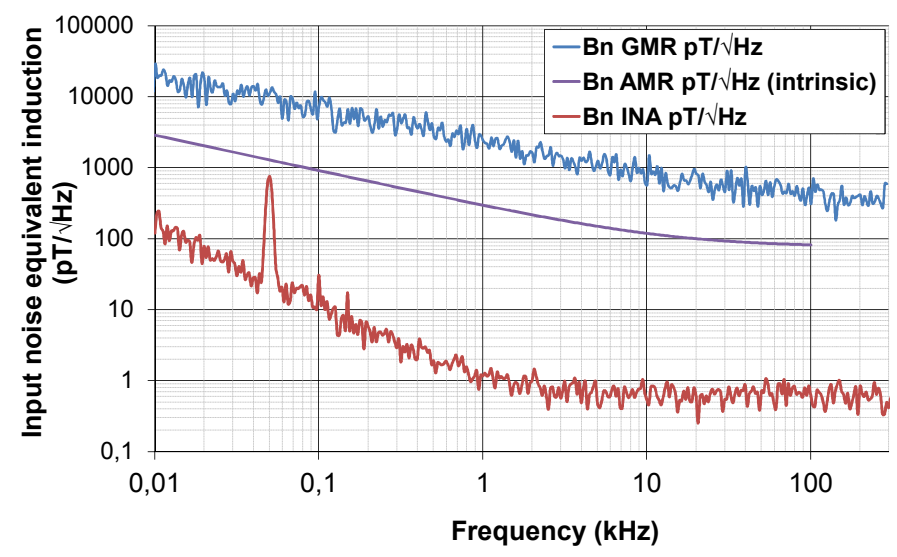

Fig. 8. Magnetic sensitivity of search coils based magnetometers using transconductance amplifier based on GMR Bridge, AMR bridge or instrumentation amplifier (INA 163). The curve for AMR bridge is deduced from the model of Fig.7 for which the noise due to the conditionning units was removed. Search coils operating in flux mode and with a transfert of $28 \mathrm{~A} / \mathrm{T}$ were considered [8].
In order to compare the magnetometers sensitivity obtained versus conditioning units, we used a compact search coil (volume less than $1 \mathrm{~cm}^{3}$ ) whose transfer was equal to $28 \mathrm{~A} / \mathrm{T}$. Measured or calculated magnetic sensitivity are given in Fig. 8. It turns out that AMR based transconductance amplifiers (better than GMR) lead to magnetometers two order of magnitude noisier than OpAmps based transconductance amplifiers. On the other hand, the MR input stage of the conditioners provides a galvanic insolation for the coil that is of primary interest. Then a significant increase of the transconductance is required. With the present AMR bridge design [6] where compensation lines are already very close to the AMR bridge, some limited improvement could be obtained by using several lines in near proximity of AMR bridge instead of a single one to increase inducting current. Another way of improvement can be investigated by reducing dimensions by a scale factor to increase input strip and AMR bridge coupling thus transimpedance. If we estimate several units gain for each way one can expect an overall improvement of at least one order of magnitude.

\section{CONCLUSION}

We achieved comparison of input referred noise of commercially available GMR and AMR based current sensors. We extracted models and estimated noise equivalent input induction of a mini search coil magnetometer using MR bridge current sensors as first stage conditioning amplifier. We found that insulated short circuit input is counterbalanced by a noisier behavior than OpAmps based conditioner. Further improvements seem achievable if CSD design is optimized as discussed.

\section{REFERENCES}

[1] M. Timofeeva, Ph'D Thesis, Université de Caen Basse Normandie, France (2012)

[2] P. Ripka, "Electric current sensors: a review", Meas. Sci. Technol. 21, 112001 (2010)

[3] NVE Data Sheet: http://www.nve.com/Downloads/ AAV003-10E Data Sheet.pdf

[4] http://www.ti.com/lit/ds/symlink/ina163.pdf

[5] Sensitec Data Sheet: http://sensitec.com.p-ad.de/upload/SENSITEC/ PDF_Downloads/Datenblatt/Sensitec_CMS2005_DSE_01.pdf

[6] G.Laimer, J.W.Kolar, "Design and experimental analysis of a DC to 1 MHz Closed Loop Magnetoresistive Current Sensor", IEEE Proceedings of Power Electronics Conference and Exposition, APEC 2005, Vol. 2, pp1288 - 1292

[7] N. Stutzke, S-E Russek, D-P. Pappas, "Low-Frequency noise measurements on commercial magnetoresistive magnetic field sensors" Journal of Applied Physics 97, 10Q107 (2005).

[8] M. Timofeeva, G. Allegre, D. Robbes, S.Flament, "Differential search coils based magnetometers: conditioning, magnetic sensitivity, spatial resolution", Sensors\&Transducers Journal, Vol 14-1, pp134-150 (2012). 